\title{
Postoperative intestinal obstruction caused by Meckel's diverticulitis after appendectomy
}

\author{
Erdal Turk $^{1 *}$, Ayca Tan², Fahri Karaca ${ }^{3}$, Yesim Edırne ${ }^{3}$, Irfan Karaca ${ }^{1}$ \\ ${ }^{1}$ Department of Pediatric Surgery, Faculty of Medicine, Izmir University, Izmir, Turkey \\ ${ }^{2}$ Clinic of Pathology, Denizli State Hospital, Denizli, Turkey \\ ${ }^{3}$ Clinic of Pediatric Surgery, Denizli State Hospital, Denizli, Turkey \\ Email: ${ }^{\text {eturk19@yahoo.de }}$
}

Received 9 October 2013; revised 5 November 2013; accepted 12 November 2013

Copyright (C) 2013 Erdal Turk et al. This is an open access article distributed under the Creative Commons Attribution License, which permits unrestricted use, distribution, and reproduction in any medium, provided the original work is properly cited.

\begin{abstract}
Surgical treatment of the complications associated with Meckel's diverticulum is widely accepted, but surgical management of an asymptomatic diverticulum found incidentally during laparotomy for other reasons is controversial. Appendectomy is the most common reason for laparotomy in many pediatric surgery clinics. The most common causes of an intestinal obstruction in the early period after appendectomy are an intra-abdominal abscess, adhesive small bowel obstruction and invagination. Obstruction due to Meckel's diverticulum is reported rarely. In this study, we present 2 cases, five and eleven years old, with an asymptomatic Meckel's diverticulum where appendectomy was performed due to acute and perforated appendicitis and obstruction developed due to Meckel's diverticulitis in the postoperative early stage. Meckel's diverticulitis must be considered in intestinal obstruction cases developing in the early stages after appendectomy although quite rare.
\end{abstract}

Keywords: Appendicitis; Meckel's Diverticulum; Intestinal Obstruction

\section{INTRODUCTION}

Whether a Meckel's diverticulum found incidentally during appendectomy should be simultaneously excised or not is a controversial issue [1,2]. The most important factors in making this decision are whether the appendix is perforated or inflamed and the macroscopic appearance of the Meckel's diverticulum [2]. Appendectomy is the main reason for laparotomy application in children and the incidence of intestinal obstruction occurring in the early period after appendectomy is $1.3 \%-8.3 \%$ with

${ }^{*}$ Corresponding author. higher rates in complicated appendicitis [3,4]. The most common causes are intra-abdominal abscess, adhesive small bowel obstruction (SBO) and invagination. The number of cases with intestinal obstruction due to Meckel's diverticulum in the early period after appendectomy is very small [5]. We present 2 pediatric cases who underwent appendectomy for acute and perforated appendicitis and then developed intestinal obstruction due to a Meckel's diverticulum in the early postoperative period.

\section{CASE 1}

Physical examination of a 5-year-old male who presented at our hospital with a history of abdominal pain and vomiting twice one day ago revealed guarding and tenderness in the right lower quadrant, while laboratory investigations showed a WBC count of $14.600 \mathrm{~mm}^{3}$ with neutrophil dominance. Abdominal ultrasonography (US) findings were consistent with acute appendicitis. The patient was operated after preparations were been completed. Acute phlegmonous appendicitis was determined during exploration and appendectomy was performed. A Meckel's diverticulum was found approximately $60 \mathrm{~cm}$ proximal to the ileocecal valve during surgery but not excised as it was not complicated. The patient was fed orally on the first postoperative day and discharged without problems on the second day. However, the case again presented at the emergency department on the fifth postoperative day with symptoms of loss of appetite, abdominal distension and bilious vomiting that had started 1 day ago. His physical examination revealed abdominal distension and tenderness. The standing direct standing abdominal X-ray showed air-fluid levels (Figure 1(A)) and the abdominal US findings were in accordance with ileus due to Meckel's diverticulum. The patient was operated on and intestinal obstruction due to 
Meckel's diverticulum was found (Figure 1(B)). Approximately $10 \mathrm{~cm}$ of the small intestine including the Meckel's diverticulum was resected and bowel integrity was ensured with an end-to-end anastomosis. The patient had no postoperative problems and was discharged on the fourth day after recovery. Histopathological examination showed acute inflammation at the crypt bases with mucosal erosions in the appendix and acute inflammation of the serosal surface of the Meckel's diverticulum (Figures 2(A) and (B)).

\section{CASE 2}

An 11-year-old male patient had presented at another hospital with abdominal pain, vomiting and fever that started 4 days ago and antibiotic and analgesic treatment had been started. The patient presented at our emergency department when his symptoms did not recover. Widespread guard and tenderness were present in the right lower abdominal quadrant. WBC count was $21.800 \mathrm{~mm}^{3}$. Abdominal US findings were consistent with perforated appendicitis. After preoperative preparations were completed, the patient was taken to surgery. Appendicitis and localized peritonitis were determined and appendectomy and drainage performed. A Meckel's diverticulum was not searched for intraoperatively. The case was fed orally on the post-operative second day and discharge was planned on the fifth day but a bad smelling and purulent secretion started coming from his drain together with symptoms of abdominal distention and bilious vomiting. The patient had air-fluid levels in direct standing abdominal X-ray and his abdominal US showed a possible intra-abdominal abscess. Conservative follow-up was started. However, the patient's symptoms increased and his clinical picture deteriorated. He was taken to surgery on the postoperative seventh day. During exploration, $600 \mathrm{cc}$ of bad smelling and purulent fluid was drained from the abdomen. The appendectomy area was clear but the cecum and small intestine appeared edematous. A Meckel's diverticulum and ileus due to the diverticulum were found approximately $60 \mathrm{~cm}$ proximal to the ileocecal valve. Approximately $15 \mathrm{~cm}$ of small intestine including the Meckel's diverticulum was resected and bowel integrity was ensured with an end-to-end anastomosis. The patient was fed orally on the fourth postoperative day, the drain was removed on the fifth day and he was discharged without problems on the sixth day. Histopathological examinations revealed submucosal and
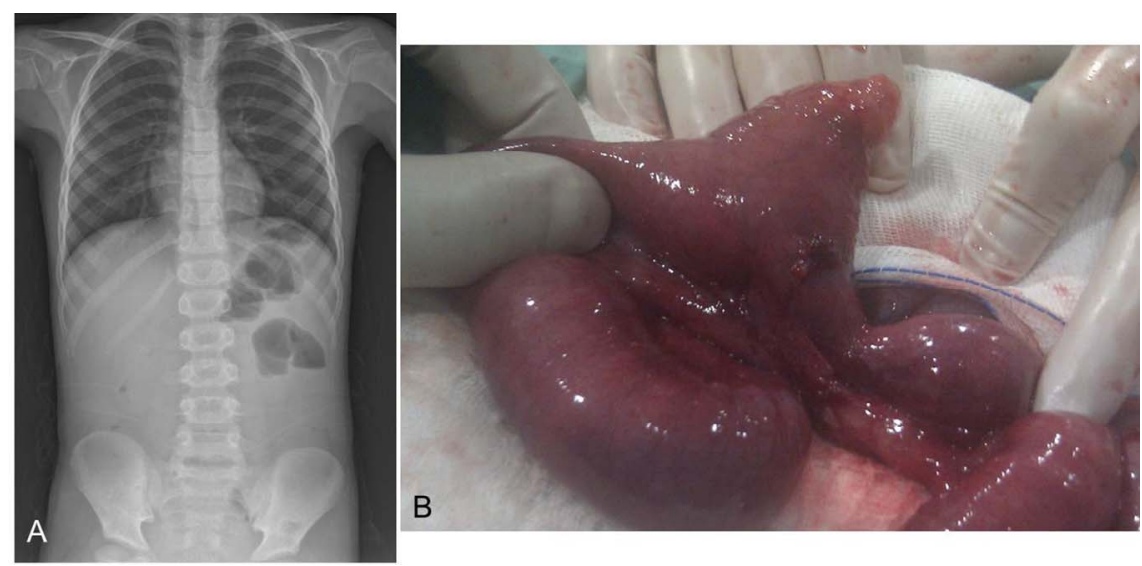

Figure 1. (A) Air-fluid levels indicating obstruction in the direct abdominal film of the first case and (B) macroscopic appearance of the Meckel's diverticulum during surgery.

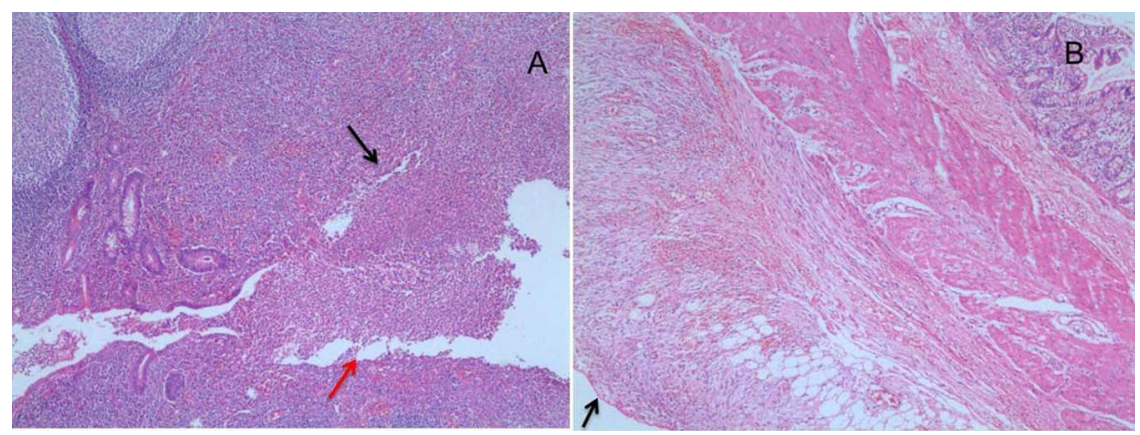

Figure 2. (A) Acute inflammation (black arrow) at the crypt bases with mucosal erosions (red arrow), $(\mathrm{H} \& \mathrm{E} \times 40)$; (B) Acute inflammation of the serosal surface of the diverticule (arrow), $(\mathrm{H} \& \mathrm{E} \times 40)$. 


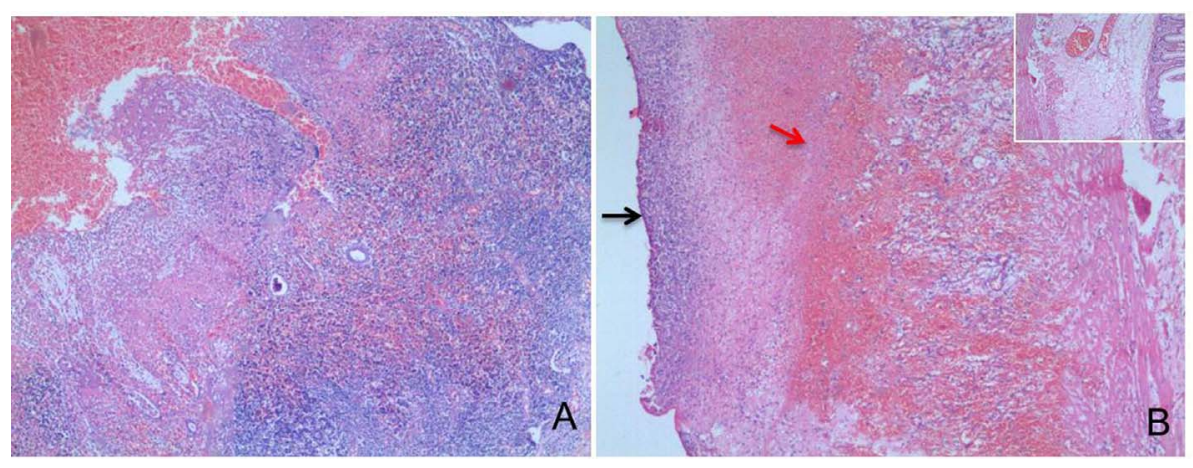

Figure 3. (A) Mucosal, submucosal and mural abscess formation and extensive mucosal ulceration, (H\&E $\times 40)$; (B) Extensive acute inflammation (black arrow) and congestion (red arrow) of serosal and subserozal area, the mucosal surface of the diverticule, $(\mathrm{H} \& \mathrm{E} \times 40)$.

mural abscess formation and extensive mucosal ulceration in the appendix and extensive acute inflammation and congestion of the serosal and subserosal area and mucosal surface of the diverticulum (Figures 3(A) and (B)).

\section{DISCUSSION}

Surgical treatment of the complications associated with Meckel's diverticulum is widely accepted, but surgical management of an asymptomatic diverticulum found incidentally during laparotomy for other reasons is controversial [6,7]. What complications a Meckel diverticulum discovered by chance will cause in a lifetime are not known and the possibility of a complication is $4.2 \%$ $6.4 \%$ [6]. Mortality in elective diverticulectomy is close to zero and mortality in complicated cases is $1 \%-10 \%$ $[6,8]$. The generally accepted approach is neither to search for a diverticulum nor to excise the diverticulum if it contains ectopic mucosa or the appendix shows lowgrade inflammation and to leave it in place in case of a perforated or gangrenous appendicitis presence but the experience and preference of the operating surgeon are an important factor [2]. The two cases presented in this study are relevant to this discussion. The first case is a typical appendicitis case determined in the early period. An uncomplicated Meckel's diverticulum was discovered intraoperatively but not removed in the belief that the pathology explaining the acute abdomen had already been detected. The second case had perforated appendicitis and presented late after using antibiotics and analgesics. The perforation was restricted to a local area, possibly because he was on medication, and the patient underwent appendectomy and drainage but a diverticulum was not searched.

The most common reason for laparotomy in many pediatric surgery clinics is appendectomy [3]. The most important causes of obstruction symptoms such as abdominal distension and bilious vomiting in the early period after an appendectomy are intra-abdominal abscess, adhesive SBO and invagination [3]. Paralytic ileus sec- ondary to perforated appendicitis may be expected to last 3 to 5 days. Prolonged bowel dysfunction or evidence of obstruction developing after temporary recovery warrants initial conservative management with nasogastric suction, fluid replacement, and possible total parenteral nutrition. Exploration may be inevitable, and the time between appendectomy and repeat laparotomy for the mechanical SBO varies between 5 days and 5 years depending on the patient's clinical picture [3]. The signs of obstruction started at the postoperative fourth day in the first case and indicated possible paralytic ileus, intestinal adhesion, or diverticulitis as we were already aware of the presence of a Meckel's diverticulum. We had no knowledge of a diverticulum in our second case, and the obstruction findings and drainage of purulent fluid after appendectomy first made us consider an intra-abdominal abscess. The rapid deterioration of the findings and hemodynamic status of both patients made us quickly decide on exploration.

In conclusion, Meckel's diverticulitis should be considered as one of the possible reasons if there are obstruction findings that develop rapidly in the early period in cases where a Meckel's diverticulum was detected incidentally but not excised or when appendectomy was performed but a diverticulum was not searched.

\section{REFERENCES}

[1] Aarnio, P. and Salonen, I.S. (2000) Abdominal disorders arising from 71 Meckel's diverticulum. Annales Chırurgıae et Gynaecologiae, 89, 281-284.

[2] Ueberrueck, T., Meyer, L., Koch, A., et al. (2005) The significance of Meckel's diverticulum in appendicitis-A retrospective analysis of 233 cases. World Journal of Surgery, 29, 455-458.

http://dx.doi.org/10.1007/s00268-004-7615-x

[3] Ahlberg, G., Bergdahl, S., Rutqvist, J., et al. (1997) Mechanical small-bowel obstruction after conventional appendectomy in children. European Journal of Pediatric Surgery, 7, 13-15. 
http://dx.doi.org/10.1055/s-2008-1071041

[4] Khairy, G.A., Afzal, M.F., Murshid, K.R., et al. (2005) Post appendectomy small bowel obstruction. Saudi Medical Journal, 26, 1058-1060.

[5] Laporte, Bouchit and Forissier (1956) Postoperative obstruction caused by diverticulitis after appendectomy for acute appendicitis. Bordeaux Chirurgical, 1, 47-49

[6] Cullen, J.J., Kelly, K.A., Moir, C.R., et al. (1994) Surgical management of Meckel's diverticulum. An epidemiologic, population-based study. Annals of Surgery, 220, 564-568, discussion 568-569. http://dx.doi.org/10.1097/00000658-199410000-00014

[7] Zani, A., Eaton, S., Rees, C.M., et al. (2008) Incidentally detected Meckel diverticulum: To resect or not to resect? Annals of Surgery, 247, 276-281. http://dx.doi.org/10.1097/SLA.0b013e31815aaaf8

[8] Korkmaz, Ö., Yılmaz, H.G. and Keleş, C. (2008) Complications of Meckel's diverticulum in adults. Dicle Medical Journal, 35, 91-95. 